\title{
Predictive Analytical Model for Microblogging Data Using Asset Bubble Modelling
}

\begin{abstract}
Srinidhi Hiriyannaiah, Department of ISE, Ramaiah Institute of Technology (MSRIT), Bangalore-560054, India \&
\end{abstract} Visvesvaraya Technological University, Belagavi, Karnataka, India

Siddesh G.M., Department of ISE, Ramaiah Institute of Technology (MSRIT), Bangalore-560054, India \& Visvesvaraya Technological University, Belagavi, Karnataka, India

Srinivasa K.G., National Institute of Technical Teachers Training and Research, Chandigarh, India

\begin{abstract}
In recent days, social media plays a significant role in the ecosystem of the big data world and its different types of information. There is an emerging need for collection, monitoring, analyzing, and visualizing the different information from various social media platforms in different domains like businesses, public administration, and others. Social media acts as the representative with numerous microblogs for analytics. Predictive analytics of such microblogs provides insights into various aspects of the real-world entities. In this article, a predictive model is proposed using the tweets generated on Twitter social media. The proposed model calculates the potential of a topic in the tweets for the prediction purposes. The experiments were conducted on tweets of the regional election in India and the results are better than the existing systems. In the future, the model can be extended for analysis of information diffusion in heterogeneous systems.
\end{abstract}

\section{KEYWORDS}

Apache Storm, Asset Bubble Model, Forecasting, Information Diffusion, Sentiment Analysis, Twitter Data

\section{INTRODUCTION}

With the widespread adoption of internet and smart phones, social media plays a significant role in various areas of business and research areas with unprecedented reach to users from all walks of life (Zeng et al., 2010). There is an emerging need to collect, monitor and analyze the different types of information from social media in the areas of business, politics, public administration and consumer-decision making (Stieglitz et al., 2014). The motivation of the paper is predictive analytics based on topics in microblogs of social media that acts as a representative for real world events (political campaigns, flu propagation, etc.). Twitter is used as a microblog for evaluation of the prediction and analytics.

Twitter is an online social networking platform where users exchange the messages in the form of tweets. It can be attributed as a micro blog where users exchange information in short text form limiting to 280 characters. The limited size helps to focus on the important part of the message rather than 
reading through the entire text. It is now used as one of the popular social media tools for exchanging messages. There is a growing base of useful news and knowledge content on Twitter. Users use hash tags to give context to their tweets and opinions that can be used in data analysis for classification of tweet. The hash tags allow making tweets relevant to a particular event. The information within a tweet can be extracted into meaningful information for further analytics.

The motivation for the implementation of a predictive analytics model is based on 'topics' in microblogs representative of real-world events/phenomenon. In this paper, the prediction model is proposed by combining the information diffusion and asset bubble model topics. Users in the twitter tweet about various events. These events are represented as 'topics' in the microblog data. In several instances, since the events are of short-time duration there is no historical data available that is associated to a topic of interest. It can be in the form of short-time duration data but with no properties of a time series data. Therefore, the traditional time-series forecasting approaches are not effective for analysis and prediction.

For analysis of tweets the proposed method uses information diffusion and asset bubble model. Each tweet has an influential potential associated with it. For example, consider a tweet "The computer purchased is good, but the delivery experience was bad". The potential of this tweet can be seen as good in terms of the purchase but also bad in terms of delivery. In this paper, such short falls of the traditional techniques are identified by the proposed method. A topic in the twitter is identified and its related tweets are collected within a time interval $[0, \mathrm{~T}]$ and a time-series is built on it. The tweets are then analyzed to identify the potential of the topic using a parameterized function. The proposed approach is validated using election related data in the state of Karnataka. The predictions made by popular media articles are used to validate the claim of prediction.

\section{RELATED WORK}

Social media platforms allow collecting data and analyzing different types of information from different users and on different topics. Examples of the social media platforms include Facebook, Google+, Twitter and others. Facebook and other social media platforms data need to be statically collected and analyzed whereas twitter provides a streaming API wherein the data can be gathered in real-time. Hence, in this paper the proposed method is used with microblog twitter. Many of the experiments related to sentimental analysis choose twitter as the source since it provides the streaming service for analysis (Owen et.al). However, there are certain drawbacks of the streaming service that include scalability and processing. In order to address this issue, the proposed system includes Apache kafka as the platform for receiving the messages from the twitter. Apache kafka includes a message queuing system wherein it can collect one million messages per second (Kreps et al., 2011).

The tweets in Twitter network are generated in real-time and need to be processed as soon as the tweet is generated. The model that analyzes the tweets need to capable of handling the tweets in real-time. Thus, in the proposed method Apache storm is used as a platform for real-time distributed processing. In Apache storm a topology is created with necessary spouts and bolts that consume streams of data from the source specified. In the proposed system, the topology is configured with source as the twitter, spout emits this data into bolts where the processing of the tweets is carried out.

In (Bakshy et al., 2012) the role of social networks in information diffusion is examined with case-studies and experiments. It was evidently found that the information spreads through the network soon for those who are significantly connected than those who are not connected. The different types of ties namely strong ties and weak ties between the users in a social network was closely examined and inferred that the influence was stronger in the locations of strong ties rather than the weak ties. However, the dissemination of information occurred from the weak ties in the social media. It was evident from the analysis that most of the novel information was propagated in the social media through the weak ties. The theory of weak ties was prevalent with sentiments on the different topics in social media. The main drawback of this approach is the ties of the topics need to visualized in 
the form of graph first and then the modularity aspects of the graph need to be addressed. In this paper, the proposed system addresses this issue by identifying the potential of the topic of the tweet and then analyzes the impact of it later.

Tweets represented in the form of text usually consist of textual information as well as nontextual content such as smileys and other emotional icons. Natural language processing (NLP) plays a key role in analyzing such text with correct semantics. There are various works related to sentimental analysis of the tweets using NLP. In (Arias et al., 2013) the proposed method is based on probabilistic machine learning on streaming twitter data to perform sentiment analysis. They analysis method includes several techniques such as support vector machines (SVM), support trees, linear and logistic regression models. The most notable advantage of the method is it adopts a corpus which includes smileys such as:-), :-D, :-(. It also uses time series model which assigns varying weights to the tweets based on its proximity to the event occurrence. The shortcomings of this approach include the use of a probabilistic model which is generally not scalable since it cannot be implemented on a distributed environment. Another limitation is the requirement of a large historic training corpus in training the model, which may not be available for several events such as the elections (Andrew et al., 2013; Kiseleve \& Ryshik, 2010). A parsimonious rule-based model is proposed in (Kiseleve \& Ryshik, 2010) for sentiment analysis of social media. A rule-based model is used for computational sentimental analysis that requires less training data. This method of rules helps in analysis of stream events faster and gives a trade-off for the performance of the model. The proposed method uses this rule-based model as the base method for identifying the sentimental aspect of the tweet first before identifying the potential of a tweet.

The concept of asset price bubble formation was proposed in (Kumar \& George, 2016). The main idea is to form a model of bubbles in social context where each term has a definite meaning and several behaviours. The model was formed using a stochastic differential equation with three factors i.e. mean stable value, response and random fluctuations. These three factors lead to the formation of the bubble and different types of simulation were conducted by tuning the factors for the formation of the bubble. In (Chae et al., 2014) the asset price bubble model was used in the prediction of events based on twitter streaming data. The algorithm was implemented in three stages, to obtain an asset bubble, in which the information diffusion increases exponentially until a time $\mathrm{T}$, when the event occurs. At this instant, the outcome of the event can be predicted with a great deal of accuracy. The model was implemented for the 2016 US Presidential Elections considering the topics related to the three candidates namely Sanders, Clinton and Trump. The outcome was even verified against the actual results from Real Clear which is a US political data source. The proposed approach in this paper considers the asset price bubble model for analysis (Chae et al., 2014). However, the use of superior sentiment analysis algorithms with the streaming distributed computing approach using Apache Storm enables the proposed system to be superior, scalable and fault tolerant than the standalone asset price bubble model and sentimental analysis (Kumar \& George, 2016; Chae et al., 2014).

Public behavioral analysis is one of the key areas of research that is being studied. In the events of disaster, people respond to the events in different ways. A system of capturing these events helps in analyzing the behavior of people in different times. In (Goonetilleke et al., 2014), an attempt has been made to study the influence of events on human behaviour through visualization. The visual analysis needs a proper management of the platform for storage and analysis. In ((Singh et al., 2010), the data management and its issues are discussed for the twitter data. The perspective of storage will play an important role wherein the events need to be captured in real-time. In the proposed system, Apache storm is used as one of the key platforms for capturing the real-time events.

Microblogging and analytics is used in various areas such as stock market trading and event analysis (Oliveira et al., 2017). In this regard, the impact of microblogging and analytics is studied in (Singh et al., 2018). It highlights the importance of returns on the stock market data through analytics on microblogging data. It also helps in supply chain management industries and applications where in the events can be tracked and analyzed (Sapountzi et al., 2018). The challenges that exist with the 
microblogging and analytics is the different features available for the analysis. The feature analysis, data pre-processing, visualization is considered as one of the key challenges for the microblogging analytics (Li \& Liu, 2017; Archambault et al., 2013).

User profiling and crowd management plays an important role in the microblogging analytics. In (Gupta et al., 2014), the different types of twitter crowds are analyzed to see the impact of topic among the crowd in the twitter. Sentimental analysis is one of the key applications that will help in such in twitter crowds. A social media analytical platform is needed for analyzing such crowd sentiments that has been developed in (Cea et al., 2014). The platform is distributed and helps in analyzing the various forms of events. Social network analytics can be classified into various forms such as real-time based events (Cherichi et al., 2016), wherein the different cases of the cloud platforms for twitter analytics is discussed. Cloud computing plays an important role in performing twitter analytics.

Event classification and analytics is an important part of the analytics in microblogging sites. In (Azam et al., 2015), various methods are used for exploring the events and detection using microblogs. These methods consider only analysis of the microblogs and the study of events but the prediction. Overall, the related work in the data analytics of the microblogging data is on the analysis but not prediction. In the proposed system, the prediction of the future outcomes is studied with the analysis.

\section{PROPOSED SYSTEM}

The proposed system has been architected after analysis of various related sources as discussed in the previous section. The resulting system architecture as shown in Figure 1 which shows the various components and their interaction with other components of the system. The main components of the proposed architecture include data collection, data pre-processing and data analysis with visualization in the dashboard. These components are explored in the further sections.

\subsection{Data Collection and Pre-Processing}

The data source considered for the analysis is twitter. Twitter utilizes HTTP Streaming protocol to deliver data services through an open and streaming and connection-oriented API. Conventionally data to be delivered is sent in batches by repeating requests to users. However, a streaming API uses a single connection and new results are being sent to the app through this single connection. This

Figure 1. Architecture diagram of proposed model

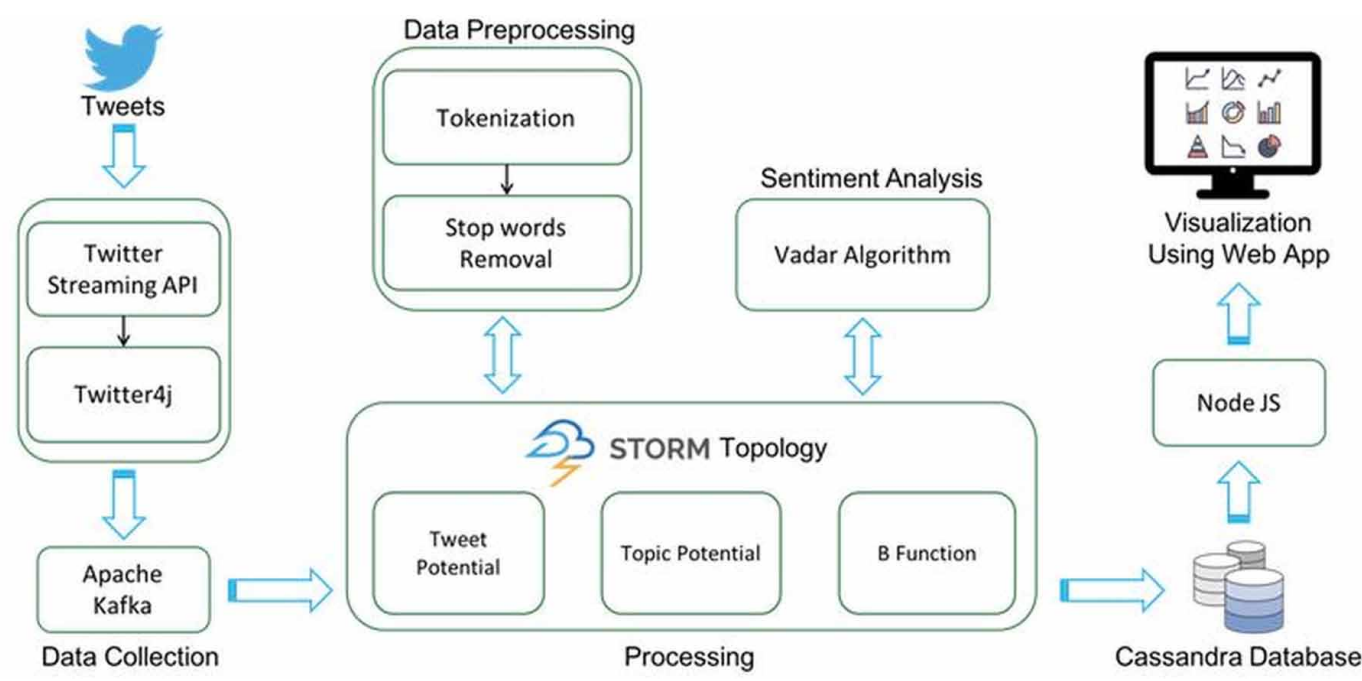


allows getting the tweets with very low-latency and high throughput. To maximize the efficiency by not losing tweets in case of slow processing, distributed messaging framework namely Apache Kafka is used. Apache Kafka, caches the tweets in a fault tolerant and distributed cluster and then streams it into Apache Storm Topology for processing. The excess tweets are cached in the Apache Kafka cluster until the Apache Storm cluster is ready to process them. This ensures that no tweet is lost even if the velocity of tweets is very high and the storm processing unit cannot keep up with it. Once the tweets are gathered it is divided into tokens by removing the stop words in the tweet. The potential of the tweet is calculated in the analysis stage as covered in the further sections.

\subsection{Data Analysis}

\subsubsection{Potential of Tweet}

The proposed algorithm calculates the potentials of the tweets and topic under consideration. A time series graph is generated from the topic potentials. Also, based on these potentials, a curve $\Delta(\mathrm{t})$ is generated using the B-Function which is our asset bubble graph. Potential of tweet $\mathrm{pz}(\mathrm{x})$ is defined as the summation of all the weights of strings in $\mathrm{L}$ if the string exists in a tweet and calculated as shown in Equation 1 where $\alpha$ represents the tokens obtained from the tweets in the data pre-processing phase. Potential of a tweet $\mathrm{p}_{\mathrm{z}}(\mathrm{x})$ is defined as writer's attitude towards a topic under consideration. It employs the topic measure $\mathrm{Z}$ representation. For each tweet, a topic $\mathrm{Z}$ is measured, which consists of 3 components $(\mathrm{X}, \mathrm{R}, \delta$ ), where $\mathrm{X}$ is a set of strings in tweets, $\mathrm{R}$ is a set of asymmetric relations which can be either 'true' or 'false' between elements of $\mathrm{L}$, and $\Pi$ is a mapping function that maps a real number with every element of $\mathrm{X}$ (the weight associated to the word). The contents of the tweet are analyzed based on the topic. The real number associated with each tweet $\mathrm{x}$ called potential of tweet, $\chi(\mathrm{x})$ and is relative to the topic Z. The algorithm for calculating the potential of the tweet is as shown in the Algorithm 1:

$$
\chi_{z}(x)=\sum_{i=1}^{k}\left(\alpha_{i}\right) I\left(\alpha_{i}\right)
$$

where:

$I\left(\alpha_{i}\right)=1$ if $\alpha_{i}$ is present in the tweet $\mathrm{x}$

$=0$ if $\alpha_{i}$ is not present in the tweet $\mathrm{x}$

Algorithm 1: Tweet Potential

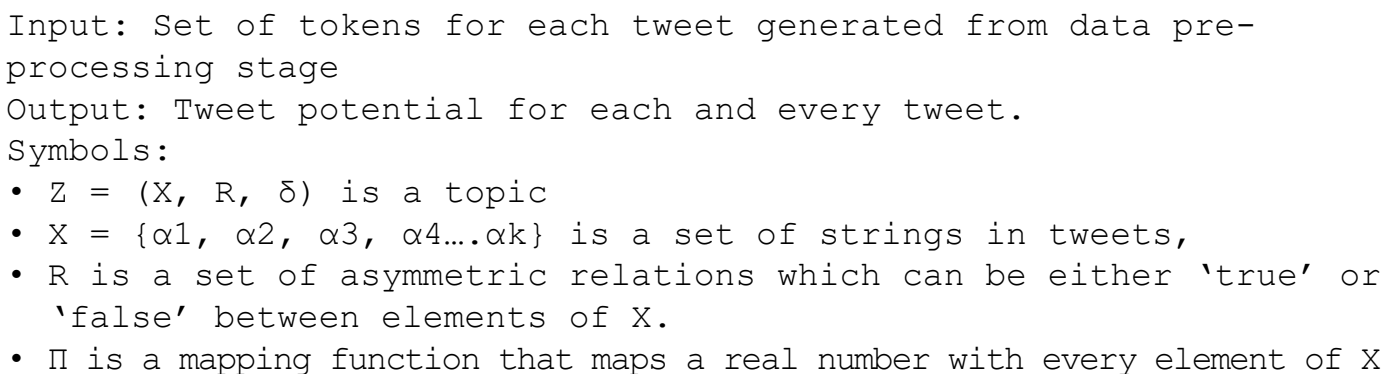


For each set of tokens for a tweet repeat until no more tokens are left Calculate $\mathrm{z}$-measure for set of tokens in a tweet using

$\chi_{z}(x)=\sum_{i=1}^{k}\left(\alpha_{i}\right) I\left(\alpha_{i}\right)$

Add it to potential of tweet, $\mathrm{p}(\mathrm{tw})$

End

\subsubsection{Topic Potential}

Once the potential of a tweet is obtained the topic based on the tweet needs to be estimated for its potential influence on others. Potential of a topic $\phi(T)$ is summation of all the potential of tweets into influence factor as calculated in Equation 2. The topic potential is general attitude of the users at a particular time t. The influence function $\Omega(1)$ is defined by the contribution of a tweet at time $t$ to the potential of the topic. The contribution of the tweet to the topic being analyzed should be capture by the influence function. The topic potential definition is very essential as it produces the time series for analysis:

$\phi(\mathrm{T})=\sum_{z=1}^{\mathrm{k}} \chi_{z}(\mathrm{x}) * \Omega\left(l_{z}\right)$

\section{Algorithm 2: Topic Potential}

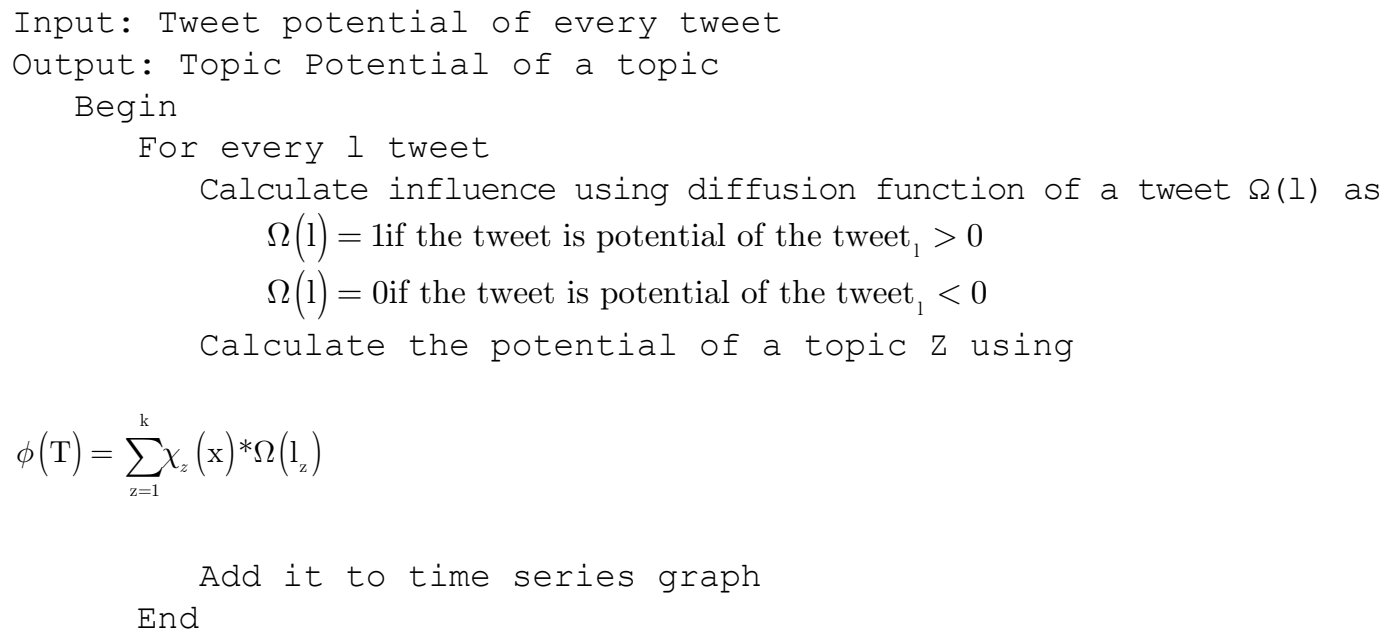

\subsubsection{Prediction}

A function (known as B-Function) is used to predict outcomes of scenarios in real time. This function sets a threshold for making a decision. Decision making is done by comparison of $\chi(\mathrm{t})$ vs $\gamma(\mathrm{t})$ in $[0, \mathrm{~T}]$ as shown in Equations 1 and 3. The growth function $\gamma(\mathrm{t})$ is exponential to $\mathrm{k}$ and linear to the parameters $\mathrm{X}$ and $\mathrm{Y}$. Here, $\mathrm{k}$ represents as the significant parameter for the growth function $\gamma(\mathrm{t})$. $\mathrm{X}$ and $\mathrm{Y}$ are used as the estimated parameters for the growth function. $\gamma(\mathrm{t})$ is used for prediction using a threshold to cross. The decision criterion is based on the threshold fixed for the growth function. Times series is defined as $\mathrm{f}([0, \mathrm{~T}])$ using the pair $(\gamma, \chi)$ where $\chi$ acts as the threshold parameter. It is 
this parameter that is used to determine the bar cross of the function. The values of $\mathrm{X}, \mathrm{Y}$ and $\mathrm{k}$ are determined as shown in the Equation 4:

$\gamma(\mathrm{t})=\mathrm{X}+\mathrm{Y}(\mathrm{T}-\mathrm{t})^{\mathrm{k}}$

where $\mathrm{t} \in[0, \mathrm{~T}], \mathrm{B}<0$ and $0<m<1$

$\mathrm{X}=\max \left(\mathrm{p}_{\mathrm{z}}(\mathrm{t})\right) \mathrm{Y}=(\min (\chi(\mathrm{t}))-\mathrm{A}) / \mathrm{T}$

\section{EXPERIMENTS AND RESULTS}

All experiments are deployed on the locally created cluster. The cluster is as shown in Figure 2. It consists of five nodes. 3 nodes are Supervisors, 1 node is zookeeper and 1 Nimbus. All the nodes had 8 gigabytes of memory and sufficient storage. These nodes are in the same local area network.

For validation of our system, tweets related to 2018 Karnataka elections were considered. The key objective is to predict the chances of the candidates winning. If the candidate is winning the sentiment associated to the candidate will increase. Candidates are considered as topics and keywords are associated to corresponding topics. Lot of experiments was conducted using Twitter data collected at different periods during the primary season. Graphical representation results are described in this section. Figure 3 shows sentiment for the topic for "BJP". The model values are plotted for 20 days with around 68,000 tweets. The BI values are between 0 and 1 for the 20-day period, this indicates that sentiments will grow after day 20.

Figure 4 shows sentiment for the topic for "Congress". The topic potential series for congress is also plotted for 20 days with around 74,000 tweets. The BI values are less to 0 for the 20 -day period. This indicates that sentiments are going to remain stagnant or even decrease after day 20.

For JDS, another major party in Karnataka elections, the number of tweets collected were very low to do the required analysis for the 20-day period. Therefore, ignored in our analysis. It is also

Figure 2. Cluster architecture for the experiment

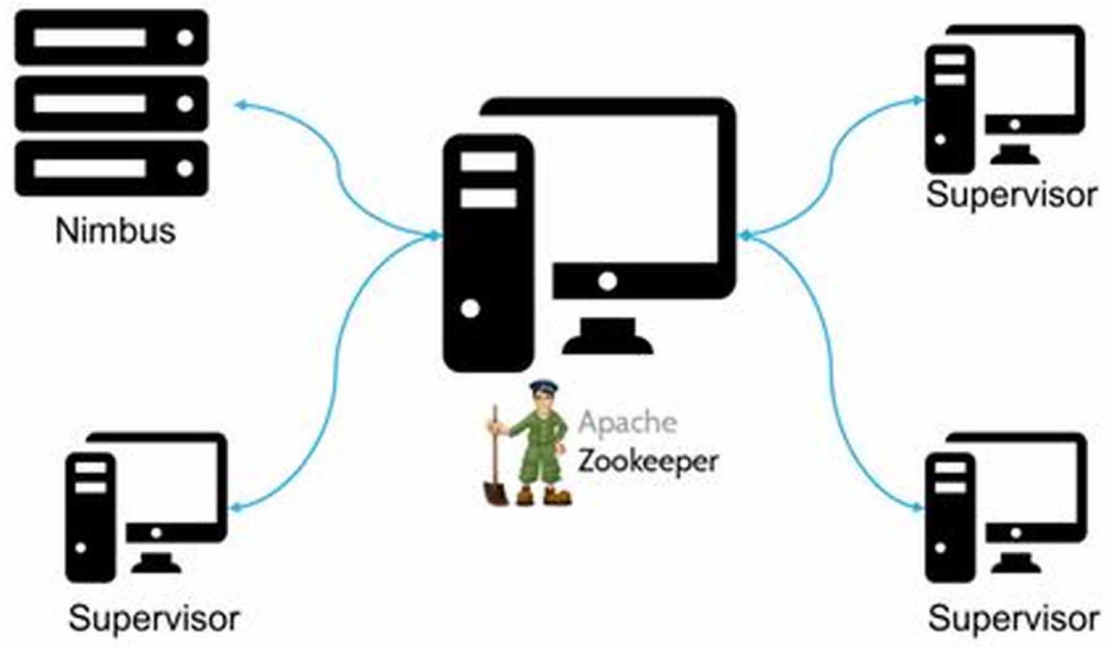


Figure 3. Topic potential time series for BJP

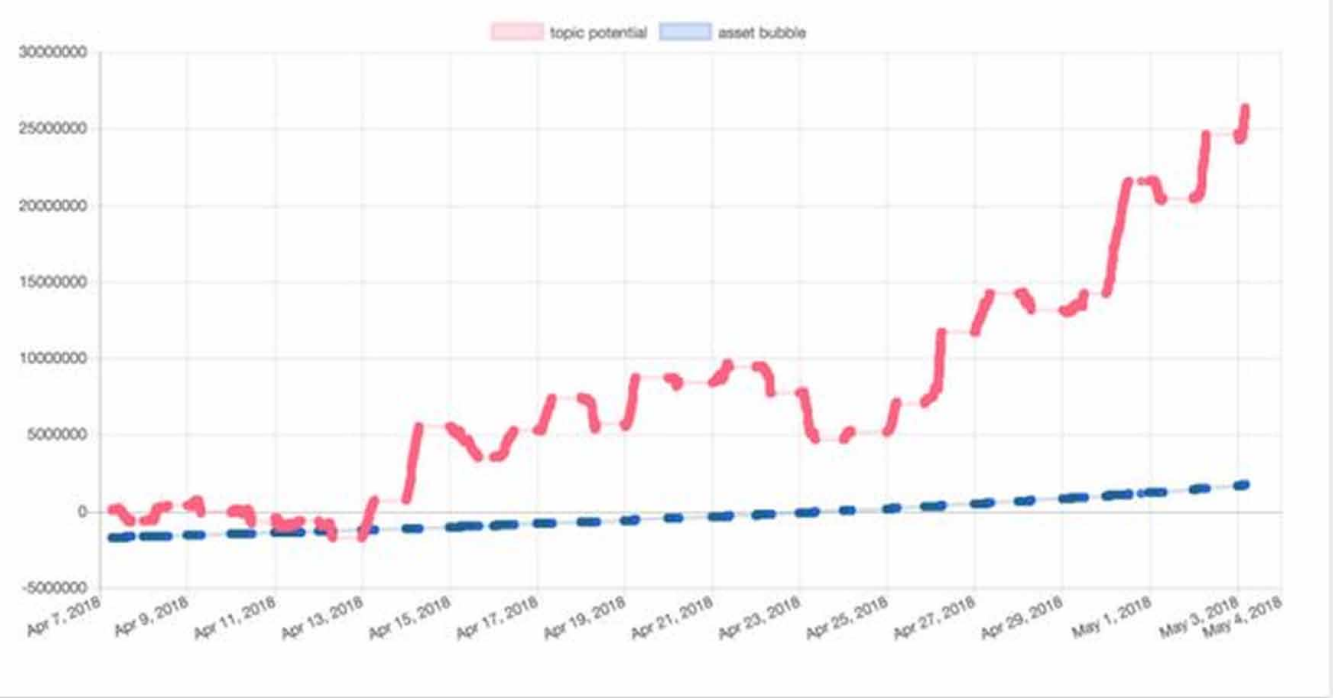

Figure 4. Topic potential time series for congress

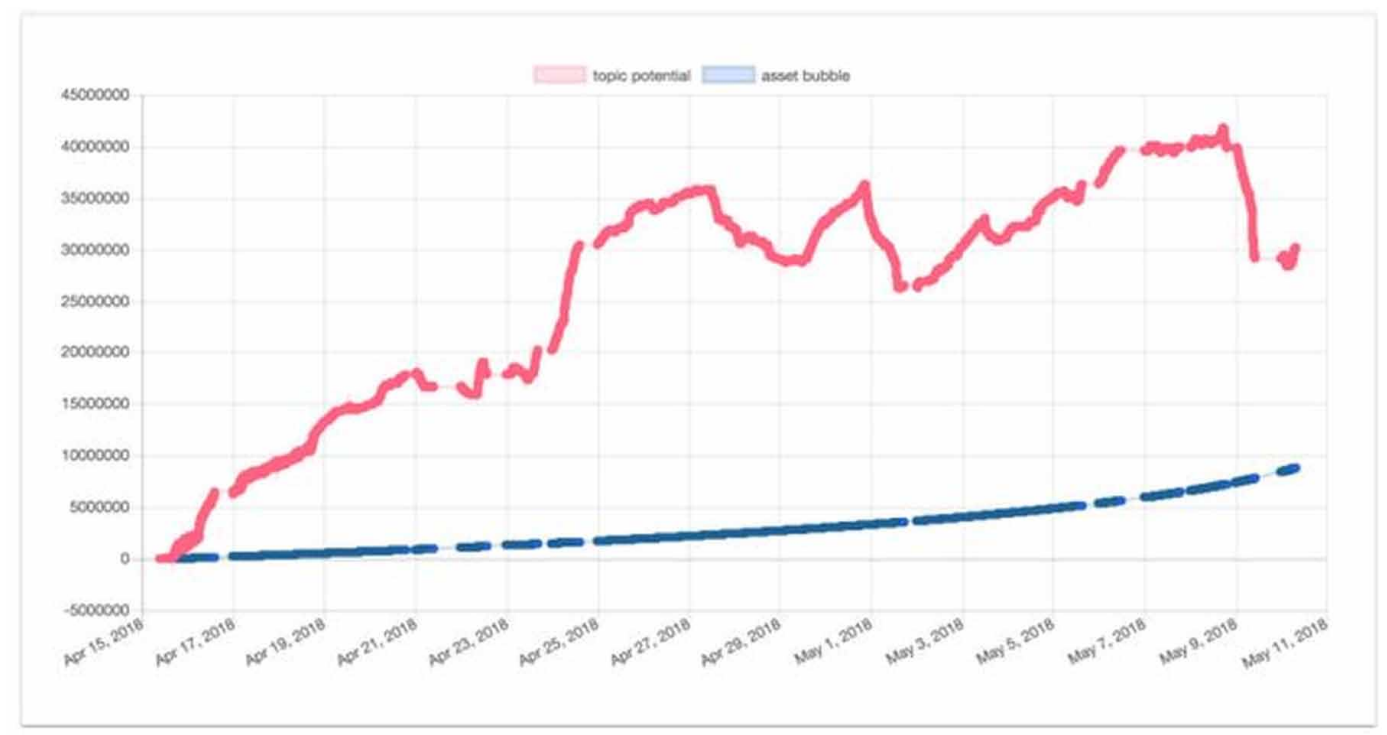

noted that the Bar Index values for both parties are very close to each other, indicating a very close election result with neither having a large margin for victory. This prediction is in line with predictions from major media agencies.

\section{CONCLUSION}

Microblogging websites have become a popular networking platform wherein the data can be used for various analytical purposes and obtain insights out of it. The paper proposed a model based on 
the asset bubble model for predicting the events in a microblogging site using election data as an example. The proposed system has been integrated with Apache storm to provide better insights into the events in the microblogging site with suitable visualization for analysis. The results of the proposed system shows that the bar index value of the asset bubble model helps in predicting the future outcomes of the events for data related to micro blogging sites. In future, the proposed system can be extended to heterogeneous ecosystem of data with analysis on different parameters such as real-time visualization of the outcomes.

\section{ACKNOWLEDGMENT}

This research was supported by Ramaiah Institute of Technology (MSRIT), Bangalore-560054 and Visvesvaraya Technological University, Jnana Sangama, Belagavi -590018. 


\section{REFERENCES}

Archambault, D., Greene, D., \& Cunningham, P. (2013). Twittercrowds: Techniques for exploring topic and sentiment in microblogging data.

Arias, M., Arratia, A., \& Xuriguera, R. (2013). Forecasting with twitter data. ACM Transactions on Intelligent Systems and Technology, 5(1), 8. doi:10.1145/2542182.2542190

Azam, N., Abulaish, M., \& Haldar, N. A. H. (2015, November). Twitter data mining for events classification and analysis. Proceedings of the 2015 Second International Conference on Soft Computing and Machine Intelligence (ISCMI) (pp. 79-83). IEEE. doi:10.1109/ISCMI.2015.33

Bakshy, E., Rosenn, I., Marlow, C., \& Adamic, L. (2012). The role of social networks in information diffusion. Proceedings of the 21 st international conference on World Wide Web (pp. 519-528). ACM. doi: $10.1145 / 2187836.2187907$

Cea, D., Nin, J., Tous, R., Torres, J., \& Ayguadé, E. (2014, October). Towards the cloudification of the social networks analytics. Proceedings of the International Conference on Modeling Decisions for Artificial Intelligence (pp. 192-203). Springer. doi:10.1007/978-3-319-12054-6_17

Chae, J., Thom, D., Jang, Y., Kim, S., Ertl, T., \& Ebert, D. S. (2014). Public behavior response analysis in disaster events utilizing visual analytics of microblog data. Computers \& Graphics, 38, 51-60. doi:10.1016/j. cag.2013.10.008

Cherichi, S., \& Faiz, R. (2016). Big data analysis for event detection in microblogs. In Recent Developments in Intelligent Information and Database Systems (pp. 309-319). Springer. doi:10.1007/978-3-319-31277-4_27

Goonetilleke, O., Sellis, T., Zhang, X., \& Sathe, S. (2014). Twitter analytics: A big data management perspective. ACM SIGKDD Explorations Newsletter, 16(1), 11-20. doi:10.1145/2674026.2674029

Gupta, M., Li, R., \& Chang, K. C. C. (2014, April). Towards a social media analytics platform: event detection and user profiling for twitter. Proceedings of the 23rd International Conference on World Wide Web (pp. 193194). ACM. doi: $10.1145 / 2567948.2577264$

Kiselev, A., \& Ryzhik, L. (2010). A simple model for asset price bubble formation and collapse.

Kreps, J., Narkhede, N., \& Rao, J. (2011). Kafka: A distributed messaging system for log processing. Proceedings of the NetDB (pp. 1-7). Academic Press.

Kumar, A.K., \& George, K.M. (2016, December). Application of an Asset Bubble Model to Microblog Data Analytics. Proceedings of the 2016 IEEE 23rd International Conference on High Performance Computing Workshops (HiPCW) (pp. 19-27). IEEE.

Li, J., \& Liu, H. (2017). Challenges of feature selection for big data analytics. IEEE Intelligent Systems, 32(2), 9-15. doi:10.1109/MIS.2017.38

Oliveira, N., Cortez, P., \& Areal, N. (2017). The impact of microblogging data for stock market prediction: Using Twitter to predict returns, volatility, trading volume and survey sentiment indices. Expert Systems with Applications, 73, 125-144. doi:10.1016/j.eswa.2016.12.036

Sapountzi, A., \& Psannis, K. E. (2018). Social networking data analysis tools \& challenges. Future Generation Computer Systems, 86, 893-913. doi:10.1016/j.future.2016.10.019

Singh, V., Gao, M., \& Jain, R. (2010). Event analytics on microblogs.

Stieglitz, S., Dang-Xuan, L., Bruns, A., \& Neuberger, C. (2014). Social media analytics. Business \& Information Systems Engineering, 6(2), 89-96. doi:10.1007/s12599-014-0315-7

Tapia, A. H., Moore, K. A., \& Johnson, N. J. (2013). Beyond the trustworthy tweet: A deeper understanding of microblogged data use by disaster response and humanitarian relief organizations. Proceedings of ISCRAM. Academic Press.

Zeng, D., Chen, H., Lusch, R., \& Li, S. H. (2010). Social media analytics and intelligence. IEEE Intelligent Systems, 25(6), 13-16. doi:10.1109/MIS.2010.151 
Srinidhi Hiriyannaiah is a research scholar at VTU. He received his Master of Technology in Software Engineering from M.S. Ramaiah Institute of Technology, Bengaluru (VTU). He is currently working as an Assistant Professor in the Department of Computer Science and Engineering at M.S. Ramaiah Institute of Technology, Bengaluru. He previously worked at IBM India Software Labs, Bengaluru. His main area of interest includes studies related to parallel computing, big data and its applications, information management, and software engineering for education.

Siddesh G. M. is currently working as an Associate Professor at the Department of Information Science \& Engineering, Ramaiah Institute of Technology, Bangalore. He has published number of research papers in international journals and conferences. His research interests include Data analytics, distributed computing, grid/ cloud computing, and loT.

Srinivasa K.G. is working as a Professor at National Institute of Technical Teachers Training \& Research, Chandigarh, India and served as the principal investigator for many projects funded by UGC, DRDO, and DST. He has been a recipient of several awards, published more than 100 research papers, and authored two books. His research areas include data mining, machine learning, and cloud computing. He received his PhD in Computer Science and Engineering from Bangalore University. 